\title{
Global Burden of Paediatric Neurological Disorders
}

Charles Richard Newton

Cheryl and Reece Scott Professor of Psychiatry

University of Oxford

United Kingdom

\begin{abstract}
Neurological conditions in children represent a significant proportion of the global burden of disease, since they contribute to premature mortality and years lived with disability. The burden of neurological conditions, as measured by the total Disability Adjusted Live Years (DALYs) has decreased significantly over the last 25 years (1990-2015), mainly due to the reduction in the mortality, as the Years Lived with Disability (YLD) has increased slightly. However in some regions of the world, notably South Asia and sub-Saharan Africa, the burden remains high, driven by the high incidence of prematurity, neonatal encephalopathy and infections of the central nervous system.
\end{abstract}




\section{Introduction}

The burden of disease is the impact of a health condition, measured by epidemiological methods, quality of death and/or financial costs. It allows comparisons of diseases and conditions between the sexes, different age groups, geographical areas and chart secular trends. It is used for advocacy, identifying high-risk populations, prioritizing actions in health, planning preventive measures, assessing healthcare systems and interventions. Neurological disorders in children have received relatively little attention in the global burden of disease, but given their incidence and years lived with a disability, these conditions are likely to have a significant contribution to the global burden of diseases (GBD).

\section{Measurement}

The standard epidemiological measures such as prevalence (i.e. the number of cases identified at a particular point or sometimes over a period i.e. period prevalence) divided by the denominator, incidence (number of new cases per unit time) and mortality provide useful data, but do not describe the full impact of neurological conditions, particularly in terms of morbidity and disability.

To provide a more encompassing measure of the impact of conditions, Disability Adjusted Life Years (DALY) were devised in the 1990s to measure the global burden of disease (GBD)(Murray \& Lopez, 1994). The DALY is a single value for a condition to facilitate comparisons between geographical area, ages and time periods. It was based upon four principles: i) inclusion of health outcomes that represent a loss of welfare; ii) characteristics of the individual affected by a health outcome are restricted to age and sex, since these demographic variables are most reliably recorded; iii) consider health outcomes equal throughout the world, i.e. a life is worth the same throughout the world; and iv) time is the unit of measurement, since it can be applied to many different conditions (Murray, 1994).

The DALY consists of two components: the years of life lost due to premature death (YLL) and the years of life living in states of poor health or disability (YLD). The YLD is derived from the number of years, the incidence (originally) and the disability weighting (DW):

YLD $=$ Years living with disability $\mathrm{x}$ incidence/prevalence $\mathrm{x}$ DW.

The incidence was originally used because: i) the method of calculating time lived with disabilities is more consistent with the method for calculating time lost due to premature mortality; ii) incidence is more sensitive to epidemiological trends and more sensitive to the impact of health interventions; iii) incidence provides more internal consistency than prevalence data (Murray, 1994). However since there is less data on the incidence of many disorders, the most recent calculations have been based on prevalence data (DALYs et al., 2015; Erskine et al., 2014).

Initially the DW were derived from expert opinion, but in the most recent calculations of DALYs, the DW have been based upon empirical data from surveys conducted five countries across the world (Salomon et al., 2012). So for example the DW for epilepsy are: treated, seizure free 0.072 (95\% confidence intervals 0.047-0.106); treated, with recent seizures $0.319(0.211-0.445)$; untreated $0.420(0.279-0.572)$ and severe $0.657(0.464-0.827)$.

One DALY can be considered as one lost year of 'healthy' life. The total DALY for a condition is simply the sum of YLL and YLD:

$$
D A L Y=Y L L+Y L D \text {. }
$$

The data can be represented as total DALYs, proportion of total DALYs (\%) for each condition or per capita, usually expressed as per 100,000 of the population. 
The Quality-Adjusted Life-Years (QALY) was introduced in the 1970s, and this measure takes into account both quantity and the quality of life. It is a measure of the life expectancy

corrected for loss of quality of that life caused by disorders. A year of normal health is given a QALY of 1 whilst a year of complete functional impairment (e.g. death) has a QALY of 0 . This measure is often used to measure the impact of medical interventions, and has not been applied to many paediatric neurological disorders, with the exception of epilepsy (Hawkins \& Scott, 2010) and head injury (Holmes, Goodacre, Stevenson, Pandor, \& Pickering, 2013).

\section{Global Burden of Disease}

There have been few publications specifically on the GBD of neurological disorders. In most papers neurological conditions were combined with mental health conditions. In 2010, mental, neurological and substance use disorders accounted for $10.4 \%$ of global DALYs, $2.3 \%$ of global YLLs and, 28.5\% of global YLDs (Whiteford, Ferrari, Degenhardt, Feigin, \& Vos, 2015). Mental disorders accounted for the largest proportion of DALYs (56.7\%), followed by neurological disorders (28.6\%) and substance use disorders (14.7\%). DALYs peaked in early adulthood for mental and substance use disorders but were more consistent across age for neurological disorders. 
In the GBD 2015 ((IHME). 2016), the DALYs of non-degenerative neurological conditions represents a significant proportion of the total DALYs in the pediatric age range (Figure 1). Overall the proportion is about $15 \%$ for neurological conditions across all age groups, but insults to the central nervous system (CNS) account for over $40 \%$ of the DALYs in neonates, mainly due to the effect of prematurity and hypoxic-ischemic encephalopathy. Neurological conditions have a greater burden than the common mental health disorders i.e. autism and ADHD.

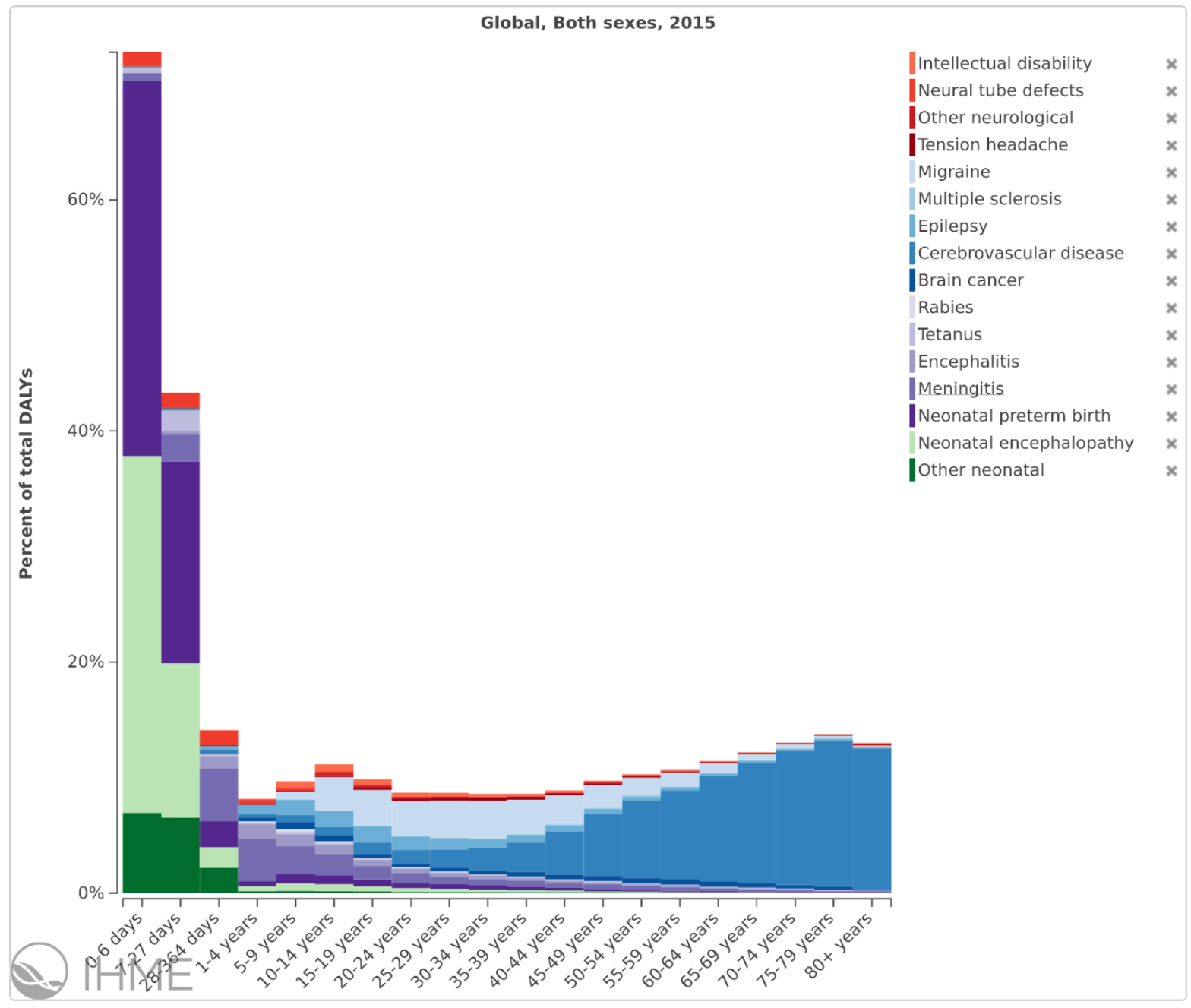

Figure 1 Contribution of non-degenerative neurological conditions to GBD as a percentage of DALYs in 2015 
Most of this burden is accounted by death, as the YLD from these disorders is under $10 \%$ (Figure 2). In older children, CNS infections are a significant burden (Figure 1 and 2), even though these figures do not include the CNS involvement of the major infections such as HIV, malaria and tuberculosis, nor traumatic head injuries.

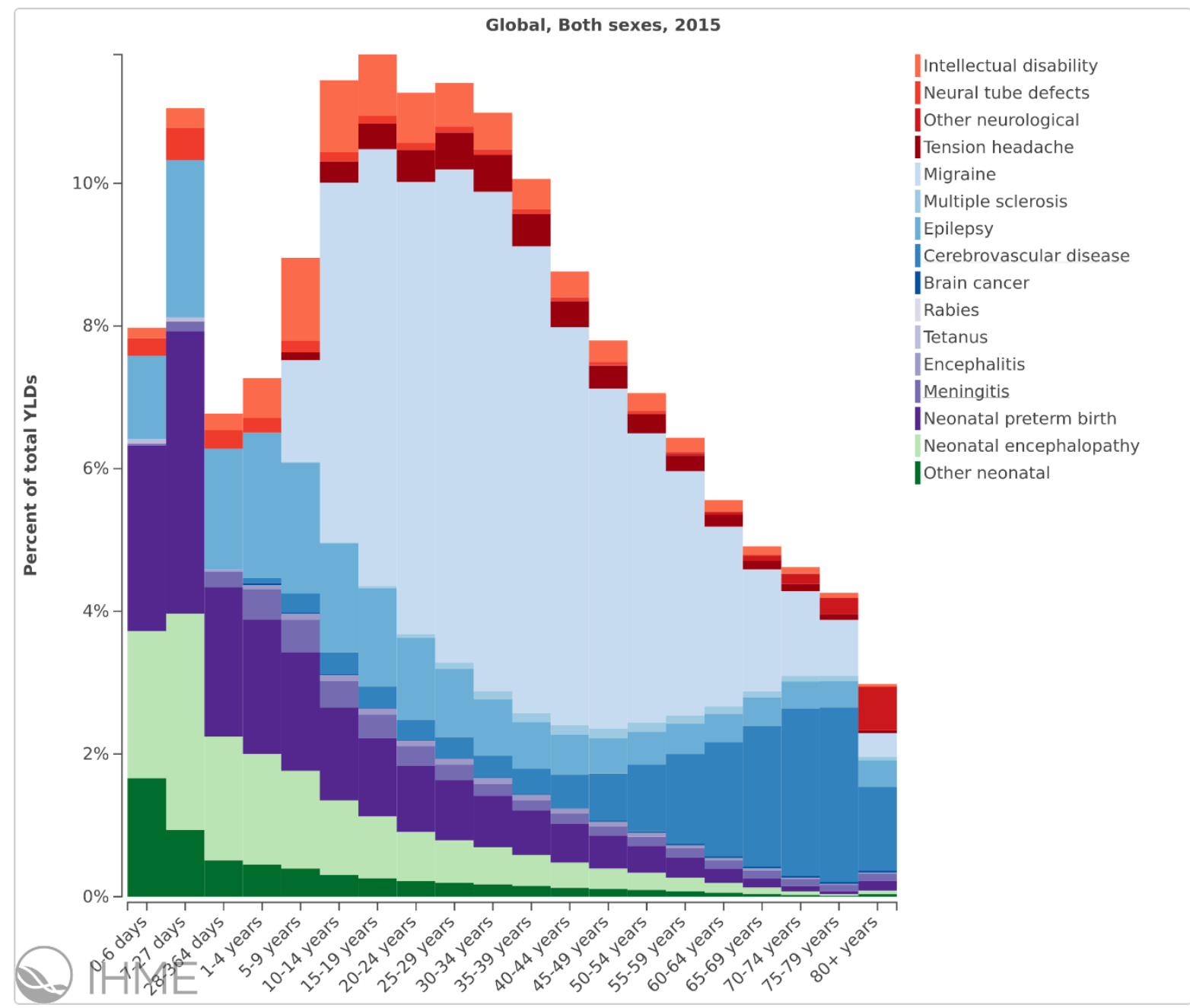

Figure 2: Contribution of non-degenerative neurological disorders to GBD as measured by YLD in 2015 in all ages 
Globally there have been significant changes in the burden of neurological conditions in children from the initial estimated in 1990 and those in 2015. The total number of DALYs attributed to neurological disorders in children has decreased, with particular marked reduction in DALYs associated with prematurity ( $57 \%$ reduction); but less with hypoxicischemic encephalopathy (23\% reduction) in the under 5 year olds (Figure 3 ).

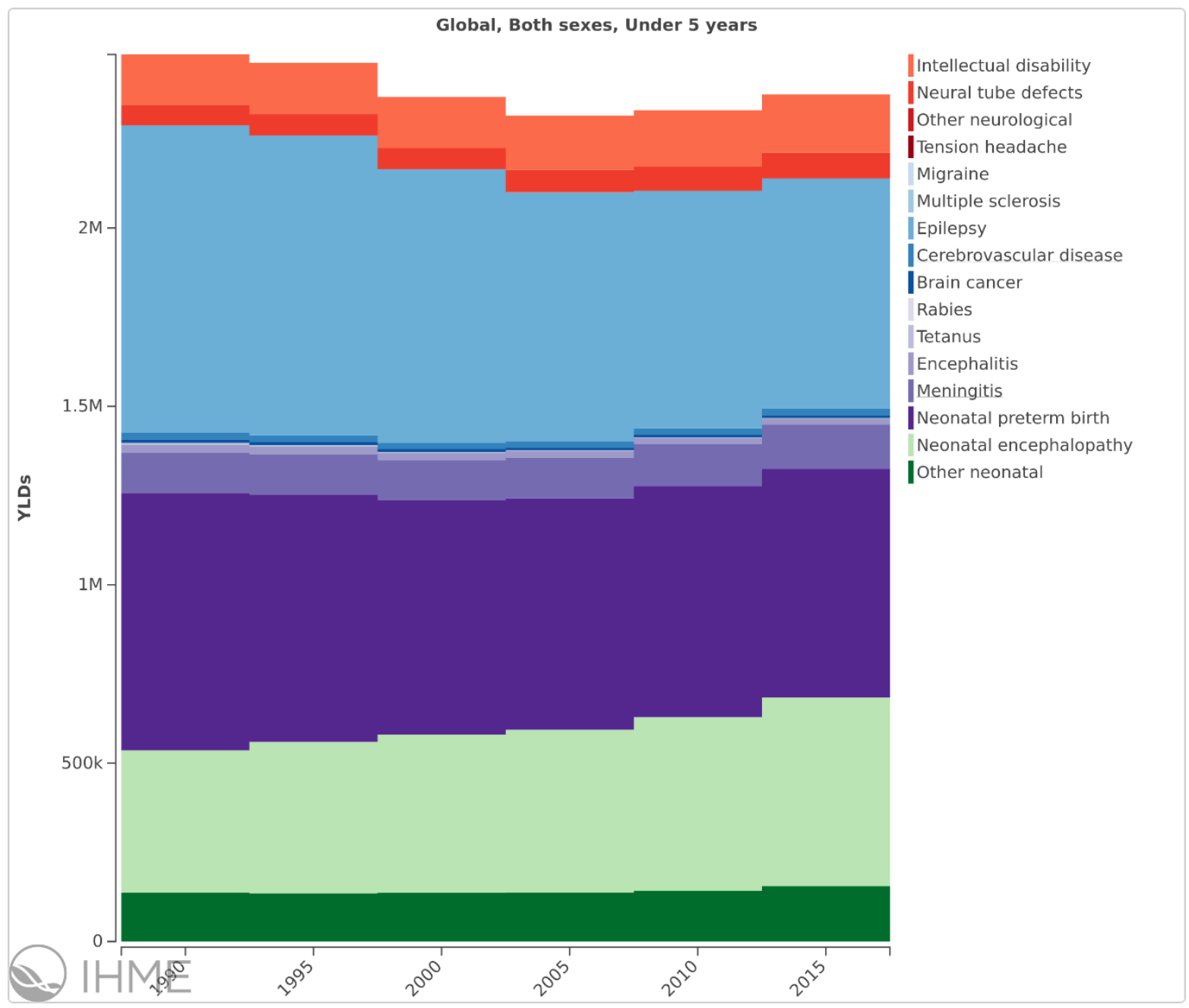

Figure 3: Years Lived with Disability of children (aged 5-14 years) with Neurological Disorders from 1990 to 2015 
In the 5-14 year olds the survival of those with neonatal encephalopathy has increased the burden from 1990 to 2015 (35\% increase), but there have been significant reduction in the burden caused by meningitis ( $28 \%$ decrease), tetanus ( $78 \%$ decrease) amongst others (Figure 4). During this twenty-five year period the total YLD for neurological disorders has increased in the 5-14 years olds, and only slightly decreased in the under 5 years.

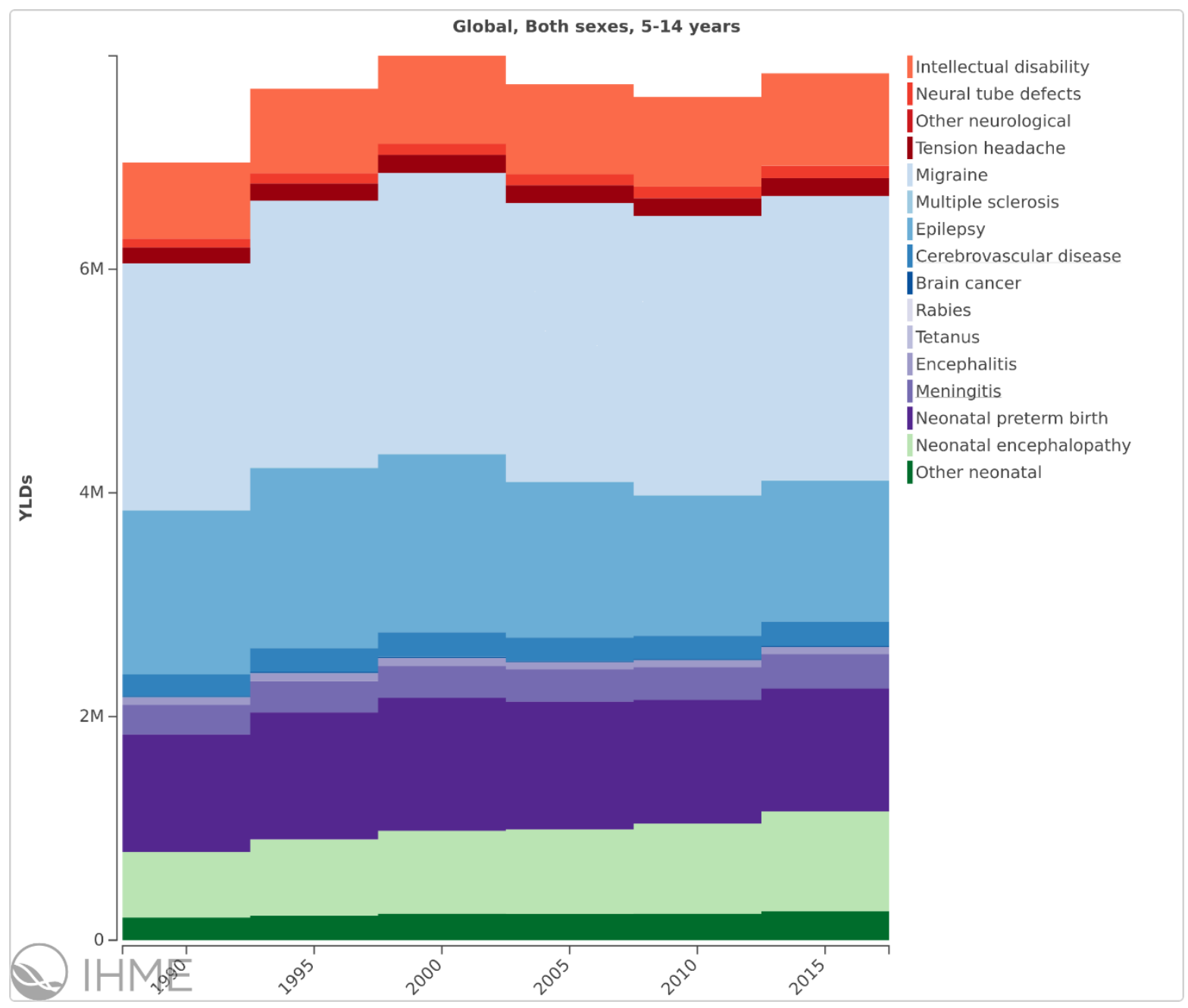

Figure 4 Years Lived with Disability of children (5-14 years) with Neurological Disorders from 1990 to 2015 
Most of the burden of neurological disorders occurs in the most populous parts of the world, namely Asia and Africa, both as total DALYs and as DALYs per capita, both for the underfives (Figure 5) and 5-14 year olds (Figure 6). There are important regional differences. In the under 5 year olds, prematurity and neonatal encephalopathy have a higher prevalence of DALYs (per 100,000) in South Asia, compared other regions of the world, including subSaharan Africa. In the 5-14 years meningitis has the largest burden in sub-Saharan Africa; whilst in South Asia, encephalitis is greater (mainly due to Japanese encephalitis).

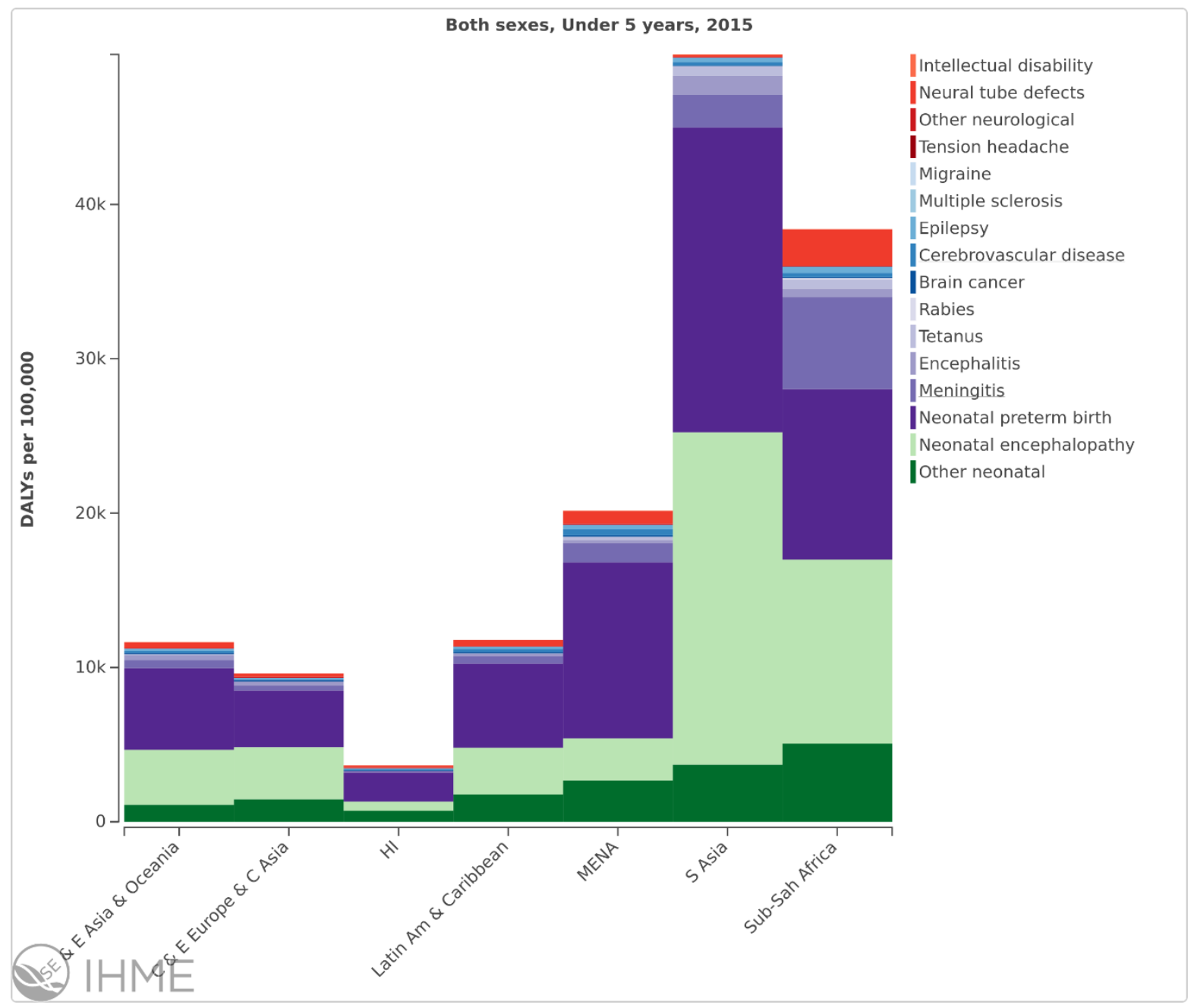

Figure 3: DALYs per 100,000 in children aged less than 5 years across the regions of the world. Key HI = High Income, MENA $=$ Mediterranean and North Africa; Sub-Sah Africa = sub-Saharan Africa . 


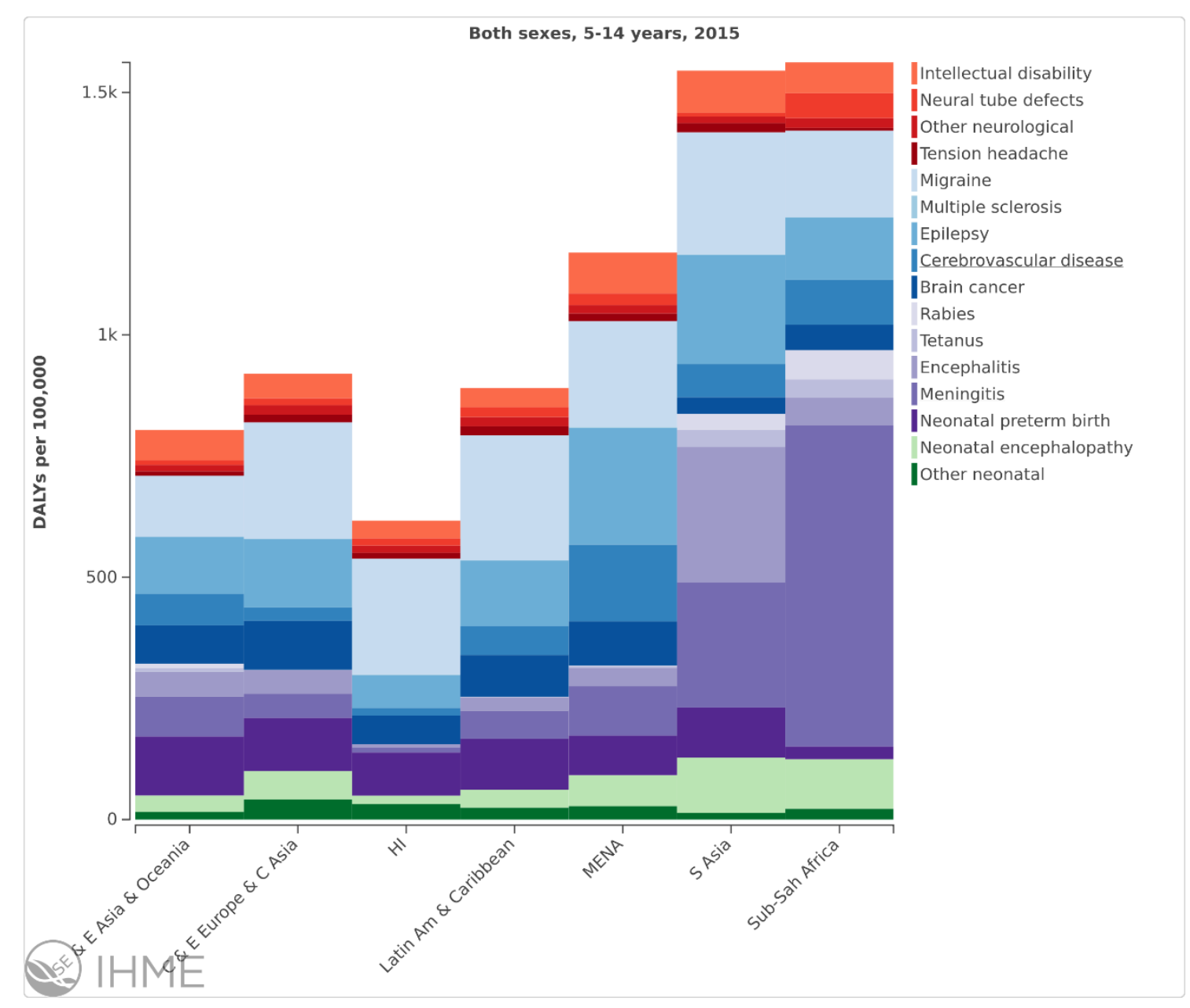

Figure 4: DALYs per 100,000 in children aged 5-14 years across the regions of the world. Key HI = High Income, MENA= Mediterranean and North Africa; Sub-Sah Africa = sub-Saharan Africa.

\section{Limitations}

The current estimates of the GBD provide a broad picture of the most common neurological conditions, but not accurate estimates of many neurological disorders e.g. many neuromuscular disorders or movement disorders (Idro, Newton, Kiguli, \& Kakooza-Mwesige, 2010). Some neurological conditions are difficult to disentangle from other major classes of diseases e.g. CNS infections from HIV and malaria, or traumatic brain injury from unintentional injuries, stroke from sickle cell disease. The data from many regions is lacking, so that the GDB extrapolates from other regions e.g. the 2015 estimate of the DALY's for autism spectrum disorders in Africa was not based on a single epidemiological study.

\section{Conclusions}

The global burden of neurological disorders in children is highest in Asia and sub-Saharan Africa. Many of the disorders originate during the perinatal period, and improved obstetrical care could significantly reduce this burden. The burden in terms of years lived with disability in children aged 5-14 years has increased significantly over the last 25 years, highlighting the need for more support in terms of expertise in the diagnosis and management of these disorders (in terms of personnel and facilities), particularly in the under-resourced areas of the world. 


\section{References}

DALYs, G. B. D., Collaborators, H., Murray, C. J., Barber, R. M., Foreman, K. J., Abbasoglu Ozgoren, A., ... Vos, T. (2015). Global, regional, and national disability-adjusted life years (DALYs) for 306 diseases and injuries and healthy life expectancy (HALE) for 188 countries, 1990-2013: quantifying the epidemiological transition. Lancet, 386(10009), 2145-2191. doi:10.1016/S0140-6736(15)61340-X

Erskine, H. E., Ferrari, A. J., Polanczyk, G. V., Moffitt, T. E., Murray, C. J., Vos, T., . . Scott, J. G. (2014). The global burden of conduct disorder and attention-deficit/hyperactivity disorder in 2010. J Child Psychol Psychiatry. doi:10.1111/jcpp.12186

Hawkins, N., \& Scott, D. A. (2010). Cost-effectiveness analysis: discount the placebo at your peril. Med Decis.Making., 30(5), 536-543.

Holmes, M. W., Goodacre, S., Stevenson, M. D., Pandor, A., \& Pickering, A. (2013). The costeffectiveness of diagnostic management strategies for children with minor head injury. Arch Dis Child, 98(12), 939-944. doi:10.1136/archdischild-2012-302820

Idro, R., Newton, C., Kiguli, S., \& Kakooza-Mwesige, A. (2010). Child neurology practice and neurological disorders in East Africa. Journal of Child Neurology, 25(4), 518-524. doi:10.1177/0883073809357792

(IHME)., I. f. H. M. a. E. (2016). GBDCompareDataVisualization. Retrieved from Available from http://vizhub.healthdata.org/gbd-compare. (Accessed [January 2017])

Murray, C. J. (1994). Quantifying the burden of disease: the technical basis for disability-adjusted life years. Bulletin of World Health Organization, 72(3), 429-445.

Murray, C. J., \& Lopez, A. D. (1994). Quantifying disability: data, methods and results. Bull World Health Organ, 72(3), 481-494.

Salomon, J. A., Vos, T., Hogan, D. R., Gagnon, M., Naghavi, M., Mokdad, A., . . Jonas, J. B. (2012). Common values in assessing health outcomes from disease and injury: disability weights measurement study for the Global Burden of Disease Study 2010. Lancet, 380(9859), 21292143. doi:10.1016/S0140-6736(12)61680-8

Whiteford, H. A., Ferrari, A. J., Degenhardt, L., Feigin, V., \& Vos, T. (2015). The global burden of mental, neurological and substance use disorders: an analysis from the Global Burden of Disease Study 2010. PLoS One, 10(2), e0116820. doi:10.1371/journal.pone.0116820 